\section{Cahiers de Narratologie}

Analyse et théorie narratives

8 | 1997

Création de l'espace et narration littéraire

\title{
Espace micro-textuel et création romanesque : réfléxion autour des épigraphes dans $E l$ reino de este mundo d'Alejo Carpentier
}

\section{Fabrice Parisot}

\section{CpenEdition}

Journals

Édition électronique

URL : http://journals.openedition.org/narratologie/11623

DOI : 10.4000/narratologie. 11623

ISSN : 1765-307X

Éditeur

LIRCES

Édition imprimée

Date de publication : 1 décembre 1997

Pagination : 295-309

ISBN : $291089746 \mathrm{X}$

ISSN : 0993-8516

Référence électronique

Fabrice Parisot, «Espace micro-textuel et création romanesque : réfléxion autour des épigraphes dans El reino de este mundo d'Alejo Carpentier », Cahiers de Narratologie [En ligne], 8 | 1997, mis en ligne le 15 décembre 2020, consulté le 25 février 2021. URL : http://journals.openedition.org/narratologie/11623 ; DOI : https://doi.org/10.4000/narratologie.11623

Ce document a été généré automatiquement le 25 février 2021.

Article L.111-1 du Code de la propriété intellectuelle. 


\title{
Espace micro-textuel et création romanesque : réfléxion autour des épigraphes dans El reino de este mundo d'Alejo Carpentier
}

\author{
Fabrice Parisot
}

1 Le type d'espace dont je voudrais vous entretenir ici est d'un genre bien particulier puisqu'il s'agit de ce qu'une partie de la critique actuelle nomme l'espace para-textuel.

2 Il est évident que mon propos ne sera pas d'étudier toutes les manifestations paratextuelles qui peuvent apparaître ou surgir dans - ou autour - d'une œuvre. Mon ambition se limitera plutôt à essayer de mettre en lumière les possibles relations que peuvent instaurer les épigraphes par rapport au texte qu'elles accompagnent de façon péri-graphique en prenant comme référent celles proposées par Alejo Carpentier dans El reino de este mundo car il nous est apparu que les quatre micro-textes qui ouvrent, jalonnent et ponctuent le récit répondent en fait à une véritable stratégie destinée à inviter le lecteur à explorer l'univers sémantique du texte dans des directions bien précises afin de le guider vers le message esthétique ou idéologique instillé, distillé tout au long du récit.

3 Je vous invite donc à présent à observer comment fonctionnent ces pièces liminaires dans l'architecture du roman et en quoi elles participent activement au processus de création romanesque.

\section{A. La première antégraphe ${ }^{1}$}

4 Située sur une page vierge avant que ne débute le roman, la première antégraphe propose au lecteur une citation tirée de l'œuvre dramatique de Lope de Vega :

Demonio :

- Licencia de entrar demando

Providencia : 
-Quién es?

Demonio :

- El Rey de Occidente.

Providencia :

- Ya sé quien eres, maldito.

Entra.

(Entra ahora)

Demonio :

- Oh Tribunal bendito

Providencia eternamente

Dónde envías a Colón

Para renovar mis daños?

No sabes que ha muchos años

que tengo allí posesión?

Lope de Vega.

5 Il s'agit d'une antégraphe allographe ${ }^{2}$ et authentique proposée sans mention de la source. Son champ d'application sémantique sera dit particularisant puisqu'elle ne s'applique en fait qu'aux huit chapitres de la première partie. Bien qu'elle soit proposée sans mention de la source, nous pouvons affirmer qu'elle est extraite de La famosa comedia de El Nuevo Mundo descubierto por Cristóbal Colón ${ }^{3}$, œuvre dont l'intention était de représenter les conséquences du choc né de la rencontre entre la culture traditionnelle et catholique de l'Espagne conquérante et celle, différente, diamétralement opposée, mais tout aussi admirable, du Nouveau Monde.

De plus, la « comedia » de Lope met en scène deux personnages d'origine haïtienne Taiparazu et Tacuana - . Or, chacun sait que El Reino de este mundo s'offre comme une chronique romancée de l'histoire convulsive de l'île d'Haïti au long de quelques soixante années. Dès lors, le choix de la pièce du Phénix comme source de l'antégraphe liminaire va fonctionner - notamment pour celui qui en aura repéré l'origine comme un révélateur de sens par omission, comme un précieux indice qui participerait au niveau de l'élaboration du sens de ce que Michel Rifaterre nomme l'intertextualité aléatoire ${ }^{4}$. En effet, le lecteur qui aura repéré l'origine de la citation avant de pénétrer dans le roman, pourra légitimement supposer par avance que les événements narrés vont se dérouler au Nouveau Monde, plus précisément en Haïti, et que la première partie va certainement offrir l'image d'une révolte du peuple noir contre le colonisateur blanc, ce qui est effectivement le cas dans le récit.

7 En outre, la présence du dramaturge Lope de Vega, et plus particulièrement de La famosa comedia de El Nuevo Mundo descubierto por Cristóbal Colón, ne se limite pas à un simple jeu de connivence culturelle proche de la devinette. Elle s'explique surtout par le fait que cette pièce est la première « comedia » écrite en Espagne sur les Amériques et qu'elle propose, de surcroît, une vision négative de l'Europe. Le choix opéré par Carpentier n'est donc pas innocent puisqu'il répond à une volonté délibérée de placer les premiers chapitres du roman sous l'égide de la première des quatre comédies américaines de Lope de Vega en tant qu'elle offre une critique négative du Vieux Monde et qu'elle suppose une idéologie subversive et révolutionnaire.

Le contenu de la citation met pour sa part en scène, dans une sorte de prologue dialogué à tendance allégorique, deux personnages antagoniques: le Démon et la Providence. Dans cet échange verbal, le Démon (Nouveau Monde) vient demander justice à la Providence (Vieux Monde) car il craint que la religion importée par Colomb ne le dépossède du monde sur lequel il règne en maître depuis la nuit des temps. 
9 Après lecture de la première partie du roman, on comprend que cette citation fonctionne en fait de façon inversée et se révèle ironique puisque le Dieu européen ne triomphera pas. En effet, on a plutôt affaire dans le récit à « une subtile synthèse de diverses cultures, de diverses croyances et de diverses religions ${ }^{5}$.»

10 Mais la présence du Démon dans le dialogue de l'antégraphe semble surtout annoncer par prolepse narrative ${ }^{6}$ extratextuelle, la geste de l'esclave noir François Mackandal considéré par ses compatriotes comme un "mandingue", c'est-à-dire comme un démon en raison de ses pouvoirs de lycanthropie :

«Además todo mandinga - era cosa sabida - ocultaba un cimarrón en potencia.

Decir mandinga era decir díscolo, revoltoso, demonio ${ }^{7}$.»

11 De plus, si le Roi d'Occident est une figure négative dans la perspective chrétienne, il acquiert au Nouveau Monde, et par là même dans le récit, des caractéristiques admirables car il représente - Mackandal et Bouckman en sont une brillante illustration - toute une série de mythologies, de croyances et de cosmogonies dans lesquelles les Noirs ont une foi inébranlable. Aussi, d'un point de vue symbolique, on peut être amené à penser que le Démon (lire Mackandal) demande la permission d'entrer dans le récit et de devenir de ce fait personnage doublement intertextuel.

Enfin, cette mise en scène entre le Démon et la Providence semble aussi annoncer a priori, ce qui sera vérifié par la suite dans le texte, tout le contexte religieux qui enveloppe le roman avec notamment l'omniprésence du vaudou ${ }^{8} \mathrm{comme}$ élément syncrétique porteur de valeurs positives et symboliques puisqu'il apparaitra dans la diégèse comme une force active en laquelle les Noirs placeront une grande partie de leurs espoirs de liberté ; une force qui sera d'ailleurs à l'origine de toutes les rebellions exposées dans le premier chapitre 9 . Par le biais de la citation liminaire, le romancier offre donc d'intéressantes pistes de lecture puisque cette première antégraphe suggère ou laisse présager le lieu des événements, la présence de personnages symboliques à tendance révolutionnaire et investis de pouvoirs démoniaques et une présentation du Nouveau Monde comme creuset de civilisations, de cultures et de croyances.

\section{B. La deuxième antégraphe}

13 La seconde citation "extratextuelle » située avant que ne débute la deuxième partie propose le «micro-texte » suivant :

... je lui dis qu'elle serait reine là-bas, qu'elle irait en palanquin, qu'une esclave serait attentive au moindre de ses mouvements pour exécuter sa volonté, qu'elle se promènerait sous les orangers en fleurs, que les serpents ne devraient lui faire aucune peur attendu qu'il n'y en avait pas aux Antilles, que les sauvages n'étaient plus à craindre, que ce n'était pas là que la broche était mise pour rôtir les gens ; enfin, $j$ 'achevai mon discours en lui disant qu'elle serait bien jolie mise en créole Madame d'Abrantés

14 Il s'agit à nouveau d'une antégraphe allographe authentique, mais donnée là encore sans mention de la source. Comme dans le cas de l'antégraphe précédente, Carpentier a omis la référence de l'œuvre d'où la citation est extraite et joue avec le degré de culture de son lecteur. Nous avons retrouvé la source de cet énoncé : il s'agit des Mémoires ou souvenirs historiques sur Napoléon ${ }^{10}$, publiés en dix volumes, entre 1831 et 1835, par Laure Permon, duchesse d'Abrantés, épouse de Jean Andoch Junot, duc d'Abrantés et général français des armées napoléoniennes. De façon schématique, on peut dire que ces 
Mémoires sont une peinture colorée, divertissante et partiale des événements de la Révolution, de l'Empire et de la Restauration auxquels elle avait assisté et même participé.

Si l'absence de la référence est ici peut-être moins pertinente que dans le cas du microtexte précédent, il n'en reste pas moins que son repérage fournit de précieuses données permettant de situer chronologiquement par avance les événements évoqués dans la seconde partie qui s'étendent de 1789 à 1802 . De plus, la présence en marge du récit d'une citation extraite d'un ouvrage à caractère historique dont l'auteur, la duchesse d'Abrantés, se pose en témoin des faits consignés tend à accréditer la véracité des événements narrés et donne un «effet de réel » historique, répondant en cela aux observations formulées par l'auteur dès le prologue de la première édition :

«/.../ el relato que va a leerse ha sido establecido sobre una documentación extremadamente rigurosa que no solamente respeta la verdad histórica de los acontecimientos, los nombres de personajes, incluso secundarios, de lugares y hasta de calles, sino que oculta bajo su aparente intempo-ralidad un minucioso cotejo de fechas y de cronologías $^{11}$.»

Il convient en outre d'observer que Carpentier a proposé cette citation dans sa langue d'origine, en l'occurrence le français, non pas pour lui conférer une aura poétique floue ou en faire une énigme mais pour suggérer par avance au lecteur que vont intervenir dans cette partie du récit des personnages historiques français ou ayant un lien étroit avec ce pays. Et effectivement, il suffira de lire les sept chapitres auxquels s'applique la citation pour y entendre parler de la Révolution Française et pour y voir apparaître le général

17 Leclerc, arrivé dans l'île en compagnie de sa femme Pauline Bonaparte, pour y mener ses campagnes et y rétablir l'ordre.

18 Signalons enfin que, venant après la citation en espagnol de Lope de Vega, l'antégraphe semble aussi établir une stratégie de l'échange, du dialogue permanent entre deux mondes, deux cultures et deux littératures.

19 Au niveau de l'énoncé, qui se présente sous la forme d'une narration à la première personne rapportant dans un dialogue au style indirect entre deux femmes la vie que mènerait l'interlocutrice en allant aux Antilles, on peut déceler une certaine ironie dans les propos tenus par Laure Permon. En effet, tous les arguments invoqués ne sont que des clichés européens sur le Nouveau Monde. Toutefois, cette vie de rêve dont parle la duchesse d'Abrantés est bien celle que Pauline Bonaparte va mener pendant un certain temps en Haiti puisque, comme le signale l'antégraphe, elle s'attachera, par exemple, les services d'un esclave attentif au moindre de ses gestes en la personne de l'eunuque noir Solimán:

«...y se aseguró los servicios de Solimán, antiguo camarero de una casa de baño ${ }^{12}$. »

D'autre part, elle possède effectivement des vêtements créoles comme en témoignent les habits emportés dans une malle sûrement d'après les conseils de Laure Permon :

«En banastas lacradasse guardaban pañuelos traídos de la isla Mauricio, los corseletes pastoriles, las faldas de muselina rayada ${ }^{13}$. »

21 Aussi peut-on constater, dans un premier temps, que l'énoncé fonctionne comme une prolepse narrative puisqu'il annonce certains événements présents dans le récit. Mais le jeu qu'instaure l'antégraphe par rapport au texte se révèle, dans un deuxième temps, plus subtil encore quand on s'aperçoit que celui-ci renvoie directement à la citation, à la personne "antégraphée » par le truchement cette fois de Pauline Bonaparte. En 
effet, celle-ci rappelle dans le récit que c'est sur les conseils de la duchesse d'Abrantés qu'elle a emporté les vêtements dont nous venons de parler :

«En banastas lacradas se guardaban pañuelos traídos de la isla Mauricio, los corseletes pastoriles, las faldas de muselina rayada que iba a estrenarse en el primer día de calor, bien instruida como lo estaba, en cuanto a las modas de la colonia, por la duquesa de Abrantès ${ }^{14}$. »

Dès lors, le lecteur comprend que la personne à laquelle s'adressait la duchesse d'Abrantés dans la citation située en hors texte n'était autre que Pauline Bonaparte qu'elle connaissait par l'intermédiaire de son mari, Jean Andoch Junot, général des armées de Napoléon au même titre que Leclerc. Aussi, les propos rapportés dans l'énoncé paraissent avoir motivé, dans le récit, le choix de la tenue vestimentaire de Pauline Bonaparte. Mais le plus intéressant peut-être, c'est que le personnage historique antégraphé devient par le biais du narrateur personnage historique allusif dans le roman.

En proposant en avant scène textuelle une antégraphé extraite d'un ouvrage à tendance historique et en propulsant sur la scène des événements celle qui l'a écrit, Carpentier offre une preuve supplémentaire que son roman est construit à partir de données exactes facilement vérifiables et identifiables. Ainsi, le réel engendre-t-il ici la fiction romanesque, laquelle, à son tour, se nourrit du réel pour prendre forme et exister pleinement. Cette seconde antégraphé loin de n'être qu'une simple orientation thématique, se veut avant tout le lieu où s'inscrivent la véracité des faits historiques suggérée par la source, le «réel-merveilleux» et surtout la dialectique Europe/ Amérique vue comme métissage culturel.

\section{La troisième antégraphe}

La troisième antégraphé, allographe et supposée authentique, établit la jonction entre la deuxième et la troisième partie du roman. Elle donne à lire un commentaire pris sur le vif :

« En todas partes, se encontraban coronas reales, de oro, entre las cuales había unas tan gruesas que apenas si podían levantarse del suelo.»

Karl Ritter, testigo del saqueo de Sans Souci

De nouveau, on peut constater que Carpentier ne divulgue pas la référence de l'œuvre dont est extraite la citation retenue. Toutefois, nous devons à Daniel Henri Pageaux ${ }^{15}$ d'avoir repéré la source exacte de la citation. Elle est extraite de l'ouvrage de Vergniaud Leconte : Henri Christophe dans l'histoire de Haïti. On a donc affaire ici à un cas très particulier d'antégraphe, puisque nous nous trouvons en présence de la citation d'une citation, ce que nous appellerons, après Antoine Compagnon ${ }^{16}$, une citation de seconde main.

Cette source - une fois repéré le jeu de transfert intertextuel - se révèle extrêmement pertinente puisqu'elle annonce et condense en fait en une sorte de résumé globalisant toute la matière narrative développée dans la troisième partie : l'histoire romancée, réélaborée de la vie d'Henri Christophe et le rôle de ce dernier dans le processus politico-historique du pays.

Par ailleurs, la présence de l'antégraphe, Karl Ritter, et surtout sa qualité de témoin oculaire semble à elle seule plus importante que le contenu même de la citation. En effet, cette précision apportée par l'auteur fonctionne déjà comme une prolepse 
narrative, comme un bref commentaire a priori d'un des faits marquants de l'histoire d'Haïti et de la vie du monarque : le sac et la destruction du palais de Sans-soucis. En quelque sorte, le «non-dit» de la référence s'oppose « au trop-dit» de l'antégraphe. Cependant ces déductions ne sont en fait possibles qu'a posteriori, c'est-à-dire après lecture de la troisième partie dans sa globalité car un lecteur innocent pourrait tout aussi bien penser légitimement que Karl Ritter fait référence ici au palais du même nom de Frédéric de Prusse qui fut selon le Siècle des Lumières un modèle de Despote Eclairé. Il y aurait donc au départ, culturellement, une sorte d'ambiguïté d'autant plus que Karl Ritter est allemand.

Signalons enfin que, comme dans le cas de l'antégraphe précédente, la présence d'un personnage historique donné à lire comme témoin effectif des faits évoqués atteste, en la redoublant, de la véracité et de l'effet de réel de la citation ainsi que de la vraisemblance de la narration. Mais ici ce n'est plus la personne antégraphée qui devient personnage allusif dans le récit, ce sont les événements narrés dans la fiction qui suscitent, d'une certaine manière, la citation et les propos de Karl Ritter. Ainsi, si la duchesse d'Abrantès entrait dans la narration par le truchement de l'antégraphe et du narrateur, Karl Ritter semble en sortir pour nous en révéler les faits survenus. Sa citation agit donc comme une mini-chronique historique présentée en décalage avec l'histoire fictive du roman mais non point avec l'Histoire d'Haïti. Elle se situe donc à la croisée des chemins entre l'histoire et l'Histoire, véritable point d'intersection qui, né d'événements vécus et rapportés fidèlement, va permettre une réélaboration historicopoétique de la part de l'écrivain.

La citation en elle-même repose presque exclusivement sur l'emblème de la royauté : la couronne. Mais ici il ne s'agit pas d'une couronne unique mais bien de plusieurs: "coronas". Cette pluralité de couronnes semble nous inviter à repérer dans le texte les différentes apparitions du mot comme si elles constituaient une sorte d'itinéraire susceptible de nous conduire vers ces ultimes couronnes d'or jetées en signe de défaite.

La première occurrence du mot nous renseigne sur les origines modestes du jeune Henri Christophe alors cuisinier et propriétaire d'une auberge à l'enseigne symbolique, La Couronne :

«Ti Noel comprendió que se hallaba en Sans Souci, la residencia predilecta del rey Henri Christophe, aquél que fuera antaño cocinero en la calle de los Españoles, dueño del albergue La Corona ${ }^{17}$.»

31 Plus tard, une fois couronné roi, le milieu ambiant dans lequel évoluera le nouveau monarque sera lui aussi placé de façon symbolique sous le signe de la couronne :

«De paso Su Majestad agarraba distraídamente una rosa blanca, recién abierta sobre los bojes que perfilaban una corona ${ }^{18}$."

32 Par ailleurs, lors de son règne, Henri Christophe s'entourera de jeunes pages dont l'un porte le nom évocateur de La Couronne :

«Allí abajo, sentados en el último peldaño de la escalera de honor, cinco negros jóvenes habían vuelto hacia él sus rostros ansiosos. En aquel instante, Christophe sintió que los amaba. Eran los bombones reales; eran Delivrance, Valentin, La Couronne, John, Bien Aimé, los africanos que el rey había comprado en un mercado de esclavos ${ }^{19} . . . »$

Et il prendra comme emblème héraldique deux lions et un Phénix couronnés :

«Se detuvo frente al dosel colorado que ostentaba sus armas. Dos leones coronados sostenían un blasón al emblema del Fénix coronado ${ }^{20}$. » 
Le parcours et l'univers du premier roi noir du Nouveau Monde sont bien marqués par la présence de l'emblème royal par excellence. Aussi, les couronnes plurielles - et polysémiques - de la citation de Karl Ritter peuvent être entendues, à un premier niveau symbolique d'interprétation, comme la synecdoque de l'ascension, de l'avènement et du règne du cuisinier de la rue des Espagnols. Mais elles vont aussi être, dans un deuxième temps, la marque de son échec, de sa destitution et de son suicide puisque, lors du pillage de Sans-souci par le peuple noir révolté, une des dernières grandes actions du tyran sera de disperser, en signe de défaite, toutes les couronnes qui sont en sa possession :

«Christophe abrió un cofre pesado, oculto por las borlas del terciopelo. Sacó un puñado de moneda de plata marcadas con sus iniciales. Luego arrojó al suelo, una tras otra varias coronas de oro macizo ${ }^{21} \ldots$ »

Ces couronnes-là renvoient inévitablement à celles de l'antégraphe en une sorte de boucle textuelle puisque la narration récupère, en cet endroit précis du récit, les propos de Karl Ritter en un subtil travail de réécriture, de réélaboration du témoignage vécu. Carpentier joue donc ici avec l'intertexte de façon intéressante puisqu'il transforme le prétexte extra-diégétique en récit diégétique et qu'il suppose une double écriture, voire une triple écriture au sujet d'un événement historique déterminant dans l'histoire de l'île.

\section{La quatrième antégraphe}

La dernière antégraphe se présente sous la forme de quatre vers composés par le dramaturge Calderón :

Miedo a estas visiones

tuve, pero luego

que he mirado a estotras

mucho más les tengo.

Calderón.

Cette citation, proposée à nouveau sans mention de la source, est allographe et authentique. Elle est extraite de la farce La muerte ${ }^{22}$ et ces mots sont prononcés à la fin de la représentation par un personnage allégorique nommé : « un caminante ». Mais rien ne semble cette fois-ci être en rapport direct dans le récit avec la source de la citation. Calderón non plus ne joue pas, par la résonance de son nom ou de son œuvre, un rôle prépondérant dans la relation interprétative prétexte/texte. Dans le cas présent, c'est plutôt l'énoncé qui fait sens.

Dans cette citation, un narrateur nous confie, à la première personne, la peur qu'il a éprouvée en étant le témoin d'horreurs survenues dans le passé (lire par déplacement symbolique les trois premières parties du roman) tout comme il exprime la crainte, plus grande encore, qu'il a ressentie devant de récentes visions, non moins terribles.

On peut alors supposer au niveau du récit qu'un personnage - présent d'un bout à l'autre du roman - va, à la première personne lui aussi, rendre compte de la peur qu'il a eue devant certaines atrocités dont il a été le témoin dans les trois premières parties et que celles qu'il va découvrir à présent lui inspirent une crainte plus grande encore. L'antégraphe semble donc fonctionner à la fois comme une sorte de mini-résumé par analepse ${ }^{23} \mathrm{du}$ texte déjà lu et comme point de vue focalisé sur les sentiments d'un personnage, par prolepse, du texte à venir. On a affaire à une citation charnière car elle 
invite indirectement le lecteur à se remémorer les événements tragiques passés, tout en laissant présager qu'il en existe de bien pires. Ces horreurs du passé, le lecteur s'en souvient, ce sont, par exemple, l'empoisonnement des animaux et des colons manigancé par le sorcier rebelle Mackandal :

«El veneno se arrastraba por la llanura del Norte, invadiendo los potreros y los establos. El hecho era que las vacas, los bueyes, los novillos, los caballos, las ovejas reventaban por centenares...Pronto se supo con espanto que el veneno había entrado en las $\operatorname{casas}^{24}$.»

Ou bien, dans la troisième partie, le despotisme exercé par le tyran Henri Christophe qui oblige son propre peuple à travailler dans des conditions inhumaines à la construction de son palais :

«Mucha gente trabajaba en esos campos bajo la vigilancia de soldados armados de látigo que, de cuando en cuando lanzaban un guijarro a un perezoso. Presos pensó Ti Noel al ver que los guardianes eran negros pero que los trabajadores también eran negros ${ }^{25}$."

41 Si ces visions ont horrifié le narrateur du roman, il en est une autre, dans la dernière partie, qui va le laisser sans voix. En effet, de retour à l'ancienne hacienda de son maître, Monsieur Lenormand de Mezy, Ti Noel se rend compte - alors qu'il croyait après la chute du tyran Christophe que la liberté était définitivement acquise par le peuple noir - que de nombreux Noirs sont sous la férule de demi-noirs, les mulâtres républicains, qui ont pris le contrôle de Saint Domingue :

«Pero, al día siguiente, observó que los Agrimenores estaban en todas partes y que unos mulatos a caballo, con camisas de cuello abierto dirigían grandes obras de

labranza y deslinde, llevadas a cabo por centenares de negros custodiados ${ }^{26}$. »

42 Ainsi, au seuil de sa vie, Ti Noel comprend-il que le peuple noir est enfermé dans un cycle qui va de l'esclavage à l'esclavage en passant par des phases de révolte et des instants de liberté. Et c'est ce cycle infernal qui constitue en définitive l'ultime vision terrifiante dont l'antégraphe semble se faire l'écho par anticipation.

\section{E. Conclusion}

Au terme de l'analyse de ces quatre antégraphes on retiendra, en tout premier lieu, le jeu de connivence culturelle instauré par le «non-dit » des sources des citations qui, en fait, en dit stratégiquement assez long sur les intentions de l'auteur puisque, dans tous les cas, on a affaire à un subtil mouvement symbolique de renvoi de texte à texte, grâce auquel le romancier transfère dans son roman, à partir d'un intéressant va-et-vient transtextuel, des personnages, des situations, des données historiques et des idéologies clefs.

Par ailleurs, au niveau des quatre énoncés, on a vu comment ils insinuent ou proposent par avance des pistes de lecture symboliques ainsi que des orientations thématiques qui seront confirmées par la suite, c'est-à-dire rétrospectivement, après lecture de chacun des différents chapitres qui structurent le récit.

Et une fois terminée la lecture du roman, on comprend que ces quatre antégraphes se chargent symboliquement d'une signification supplémentaire qui dévoile un des crédos esthétiques et idéologiques fondamentaux de la pensée du Cubain: le permanent dialogue et mélange des cultures. En effet, si dans un premier temps, la présence de deux auteurs espagnols (Lope de Vega et Calderón de la Barca), d'un auteur français (la 
duchesse d'Abrantés) et d'un géographe allemand dont la citation retenue se trouve en fait dans un ouvrage historique français, est là pour rappeler, si besoin était, que l'île d'Haïti/Saint Domingue, l'ancienne Hispaniola découverte par Christophe Colomb lors de sa première expédition, était divisée à l'époque où se déroule le roman, entre la France (zone occidentale) et l'Espagne (zone orientale), dans un second temps on comprend que la distribution des citations, deux auteurs espagnols encadrant deux citations de source française, semble être la marque évidente du permanent échange hispano-français présent d'un bout à l'autre du récit mais aussi de toute l'œuvre de Carpentier. De plus, comme le signale Daniel Henri Pageaux ${ }^{27}$, les trois coupes chronologiques retenues ici (geste de Mackandal, séjour de Pauline Bonaparte et règne d'Henri Christophe) difficilement comparables sur le plan historique et politique représentent d'une certaine façon le théâtre (les références à Lope de Vega et à Calderón de la Barca prendraient alors tout leur sens) d'une incessante interrelation et parfois d'un incroyable syncrétisme entre cultures, mais aussi d'une possible transformation des cultures dialoguant entre elles, en un mot, le "grand théâtre du monde ».

On voit donc bien en fin d'analyse comment l'espace para-textuel agit sur le texte et intercède auprès du lecteur afin de le guider stratégiquement vers l'interprétation optimale du récit.

\section{NOTES}

1. On nomme antégraphe, en accord avec l'étymologie, les citations qui ne se situent pas directement au-dessus d'une autre écriture mais avant. On dira de ces citations qu'elles sont antéposées.

2. On entend par citation allographe toute citation attribuée à un auteur différent de celui qui a écrit le roman.

3. Lope de Vega, La famosa comedia de El Nuevo Mundo descubierto por Cristóbal Colon, BAE.

4. Michel Rifaterre, «La trace de l'intertexte », in La Pensée, Paris, n² 215, oct 1980, p. 5.

5. Voir à ce sujet : Frederik de Armas, «Lope y Carpentier ", in Actas del Simposio Internacional de Estudios Hispánicos, Budapest, Akademial Kiado, 1978, p. 369.

6. Cf : Gérard Genette, Figures III, Paris Seuil, Poétique, 1972, p. 75.

7. Alejo Carpentier, El reino de este mundo, op. cit., p. 22.

8. Culte animiste des Noirs des Antilles et d'Haïti, né du mélange insolite des croyances catholiques et africaines.

9. Sur la présence et le rôle du vaudou dans le roman, vid. Ernesto Barrera, «El vodú y el sacrificio del tótem ", in Cuadernos americanos, XXXVI, CLX, 2, marz-abr 1977, pp. 148-157. Emma Speratti Piñero, "Creencias afroantillanas en El reino de este mundo ", in Nueva Revista de Filología Hispánica, XXIX, 2, 1980, pp. 574-596.

10. Laure Permon, Mémoires ou souvenirs historiques sur Napoléon, Paris, 1831-1835.

11. Alejo Carpentier, Prólogo a El reino de este mundo, op. cit., p. XV.

12. Ibid., p. 79.

13. Ibid., p. 70. 
14. Ibid., p. 77.

15. Daniel Henri Pageaux, "Alejo Carpentier devant Haïti : Le royaume de ce monde ", in Alejo Carpentier et son œuvre, Paris, L'Harmattan, 1980, pp. 131-151.

16. Antoine Compagnon, La seconde main, Paris, Seuil, Poétique, 1979.

17. Ibid., p. 90.

18. Ibid., p. 91.

19. Ibid., p. 112.

20. Ibid., p. 113.

21. Ibid.

22. Pedro Calderón de la Barca, «La muerte ", in Mojiganga, Madrid, BAE, Tome XIV, 4, 1945, p. 648.

23. On entend par analepse, un retour en arrière selon la terminologie de Gérard Genette, in Figures II et Figures III, op. cit.

24. Alejo Carpentier, El reino de este mundo, op. cit., p. 27.

25. Ibid., p. 88.

26. Ibid.,p. 138.

27. Daniel Henri Pageaux, « Alejo Carpentier devant Haïti : El reino de este mundo », op. cit., p. 148.

\section{AUTEUR}

\section{FABRICE PARISOT}

Université de Nice-Sophia Antipolis 